04

\title{
Исследования начальной фазы искрового разряда в воздухе в промежутке острие (катод)-плоскость методом лазерного зондирования
}

\author{
(C) А.А. Тренькин, К.И. Алмазова, А.Н. Белоногов, В.В. Боровков, Е.В. Горелов, И.В. Морозов, С.Ю. Харитонов
}

Российский Федеральный ядерный центр, Всероссийский научно-исследовательский институт экспериментальной физики,

607190 Саров, Россия

e-mail: trenkin@ntc.vniief.ru

Поступило в Редакцию 25 декабря 2019 г.

В окончательной редакции 14 мая 2020 г.

Принято к публикации 14 мая 2020 г.

С использованием метода лазерного зондирования исследован искровой разряд в промежутке острие (катод)-плоскость длиной $1.5 \mathrm{~mm}$ в интервале времени от момента пробоя до $200 \mathrm{~ns}$. Установлено, что разряд развивается в виде пучка микроканалов. Определена динамика пространственной структуры разряда, концентрации электронов, температуры газа в канале и скорости его радиального расширения.

Ключевые слова: искровой разряд, микроструктура, интерферометрия, концентрация электронов.

DOI: $10.21883 /$ JTF.2020.12.50119.435-19

\section{Введение}

Исследование высоковольтного импульсного разряда в плотных газах и, в частности, искрового, представляет большой интерес как с фундаментальной точки зрения, так и в связи с его широким практическим применением. Особую актуальность имеет получение экспериментальных данных о начальной фазе развития разряда, что обусловлено определенными трудностями регистрации быстропротекающих мелкомасштабных процессов.

В пионерских исследованиях искрового разряда [1-4] были получены широкие спектры экспериментальных и расчетно-теоретических данных о развитии разряда, однако они касались в основном газодинамической фазы на временах более $100 \mathrm{~ns}$.

Необходимое для исследования начальных фаз разряда пространственное и временное разрешение можно обеспечить методами лазерного зондирования. Так, в работах $[5,6]$ посредством указанных методов была исследована в том числе предпробойная фаза разряда, однако использованная методика имела пространственную разрешающую способность не лучше $10 \mu \mathrm{m}$ [6].

В работе [7] исследовалась пространственная и временная эволюция концентрации плазмы искрового разряда в воздухе с использованием интерферометра Маха-Цендера. Пространственное разрешение оптической системы регистрации составляло при этом $5 \mu \mathrm{m}$ на один пиксель.

В работах [8-10] была реализована методика исследования пространственной структуры разряда методом теневого фотографирования с пространственным разрешением $5 \mu \mathrm{m}$ на три пикселя. В этих исследованиях была зарегистрирована микроканальная структура искрового разряда в воздухе атмосферного давления в промежутке острие-плоскость на начальной стадии его развития. Установлено, что динамика структуры на временах от единиц до десятков наносекунд включает развитие каналов микронного диаметра от острия вглубь разрядного промежутка, их ветвление и расширение. Затем происходило формирование общего фронта ударной волны канала искры и его движение. Одновременно с этим на поверхности плоского электрода была зарегистрирована обусловленная разрядом эрозионная микроструктура в виде совокупности большого количества микрократеров [11]. Было показано, что описанная динамика разряда слабо зависит от параметров (материал, форма поверхности) острийного электрода. Следует отметить, что указанные работы в основном посвящены исследованию разряда, формируемого в промежутке острие-плоскость с острийным анодом.

Настоящая работа является продолжением исследований [8-10]. Здесь в дополнение к методике теневого фотографирования реализована интерференционная методика, с помощью которой получены дополнительные данные о процессах, сопровождающих искровой разряд.

\section{1. Теория и допущения}

При прохождении световой волной оптической неоднородности (в нашем случае газоразрядной плазмы) она приобретает некоторый дополнительный фазовый сдвиг по сравнению с такой же волной, распространяющейся в невозмущенной среде. На интерферограммах это выражается в виде смещения интерференционных полос.

В общем случае смещение интерференционных полос $\Delta m$ (в долях ширины полосы) связано с изменение показателя преломления $\Delta n=n-n_{0}$ возмущенной обла- 


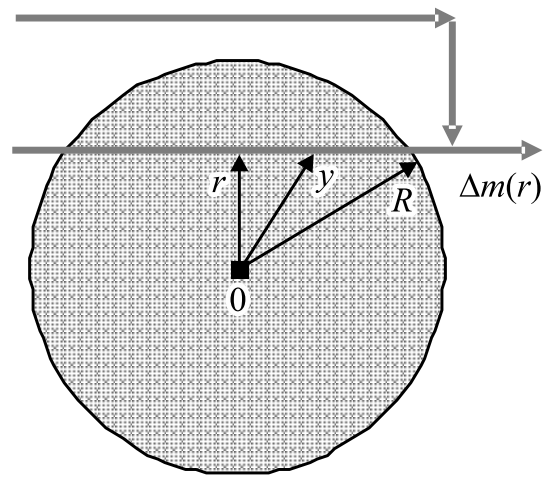

Рис. 1. К уравнению Абеля.

сти как:

$$
\Delta m=\frac{1}{\lambda} \int_{z_{1}}^{z_{2}} \Delta n d z
$$

где $z_{1}, z_{2}$ - точки входа и выхода луча из возмущенной области, $L=\left(z_{2}-z_{1}\right)$ - ее размер, $n_{0} \approx 1.0003$ - показатель преломления воздуха при атмосферном давлении, $\lambda$ - длина волны зондирующего излучения.

Для осесимметричных неоднородностей (1) переходит в интегральное уравнение Абеля:

$$
\Delta m(r)=\frac{2}{\lambda} \int_{r}^{R} \Delta n(y) \frac{y d y}{\sqrt{y^{2}-r^{2}}},
$$

где $R$ - радиус неоднородности, $r-$ расстояние от хорды до оси симметрии (рис. 1).

Выполнение обратного преобразования (2) позволяет восстановить распределение изменения показателя преломления по радиусу $\Delta n(r)$. В настоящей работе восстановление проводилось следующим образом. Поперечное сечение исследуемой неоднородности разбивалось на двадцать концентрических кольцевых зон, в каждой из которых поведение $\Delta m(r)$ задавалось ступенчатой аппроксимацией. Для определения изменения показателя преломления в каждой зоне численно решалось обращенное уравнение Абеля [12]:

$$
\Delta n_{i}=\frac{\lambda}{\pi R} \sum_{k=i}^{N-1} \Omega_{i, k} \Delta m_{k},
$$

где $\Delta n_{i}=\left(n_{i}-n_{0}\right)$ - изменение показателя преломления в $i$-ой зоне, $\Delta m_{k}-$ смещение интерференционной полосы в соответствующей зоне, $\Omega_{i, k}$ - численные коэффициенты.

В изменение показателя преломления плазмы входят следующие составляющие: изменение концентрации атомов, молекул и ионов газа, а также свободных электронов. Полагая, что наличие ионов и продуктов диссоциации не приводит к заметному изменению рефракционных свойств плазмы, выражение для показателя преломления среды для области нормальной дисперсии можно записать в виде [13]:

$$
n=1-4.46 \cdot 10^{-14} N_{e} \lambda^{2}+C_{m} N_{m}
$$

где $N_{e}$ - концентрация электронов, $N_{m}$ - концентрация частиц газа, $C_{m}$ - вклад газа в пересчете на одну частицу. Здесь в качестве единицы длины используется сантиметр.

Рассматривая только рефракцию на свободных электронах из (4), имеем

$$
N_{e}=\frac{n-1}{4.46 \cdot 10^{-14} \lambda^{2}} .
$$

Отсюда с учетом (1) для оценки среднего по длине оптического пути $L$ значения концентрации электронов $\left\langle N_{e}\right\rangle$ для используемой длины волны лазера $\lambda=5.32 \cdot 10^{-5} \mathrm{~cm}$ получаем

$$
\left\langle N_{e}\right\rangle=4.2 \cdot 10^{17} \frac{\Delta m}{L} .
$$

Следует отметить, что для указанной длины волны критическое значение концентрации плазмы составляет $4 \cdot 10^{21} \mathrm{~cm}^{-3}$, что значительно превосходит концентрации, характерные для газовых разрядов атмосферного давления. Следовательно, такая плазма будет прозрачна для излучения и его можно использовать для зондирования.

\section{2. Экспериментальная аппаратура и методика}

Схема экспериментального стенда представлена на рис. 2. Часть элементов схемы и методика теневого фотографирования подробно описаны в [8]. В общих чертах эксперименты заключались в следующем. На разрядный промежуток с генератора импульсов напряжения посредством кабельной линии подавался импульс амплитудой $25 \mathrm{kV}$ и длительность фронта по уровню 0.1-0.9

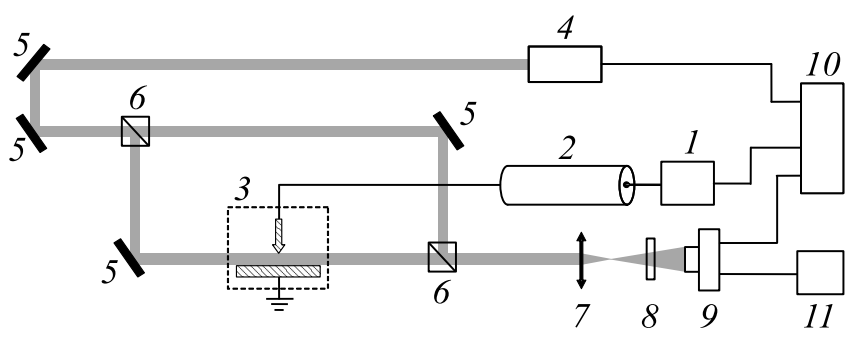

Рис. 2. Схема экспериментального стенда: 1 - генератор импульсов напряжения, 2 - кабельная линия, 3 - разрядный промежуток, 4 - источник зондирующего сигнала (лазер), 5 - поворотные зеркала, 6 - светоделительные элементы, 7 - объектив, 8 - светофильтры, 9 - электронно-оптический регистратор, 10 - блок синхронизации, $11-$ персональный компьютер. 

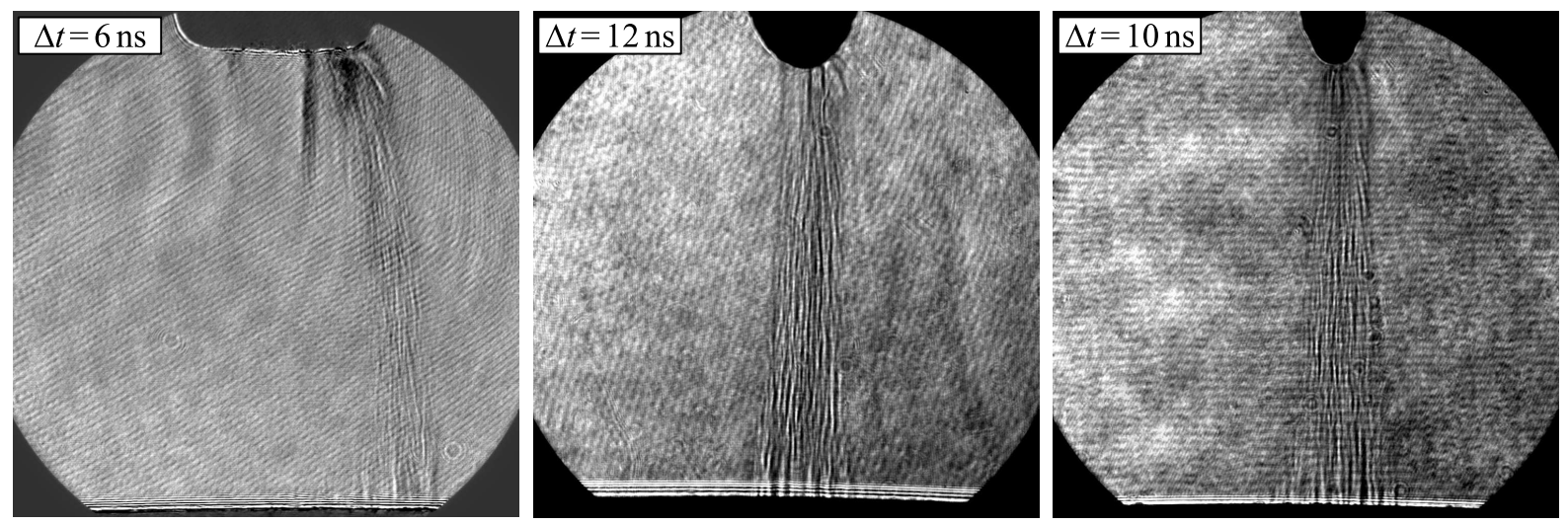

Рис. 3. Тенеграммы разряда для различных острийных электродов. На изображениях указаны моменты времени относительно $t_{b d}$. Электрод-острие находится сверху.

около 7 ns. Регистрация напряжения и тока осуществлялась на выходе генератора импульсов напряжения.

Электродная система имела геометрию „острие-плоскость“. Использовалось несколько осесимметричных острийных электродов из нержавеющей стали, отличающихся формой поверхности и подробно описанные в [10]. Полярность импульса напряжения, подаваемого на острийный электрод, - отрицательная. В большей части экспериментов применялся электрод (далее по тексту основной) длиной $19 \mathrm{~mm}$, диаметром $14 \mathrm{~mm}$ с углом при вершине $36^{\circ}$ и радиусом кривизны $0.15 \mathrm{~mm}$. В качестве плоского электрода использовался электрод, изготовленный из алюминиевого сплава, с рабочей частью, близкой по форме к шаровому сегменту диаметром $4.5 \mathrm{~cm}$ толщиной $1.5 \mathrm{~cm}$. Межэлектродный зазор составлял $1.5 \mathrm{~mm}$.

После пробоя промежутка в разрядной цепи возникал колебательный процесс с экспоненциальным затуханием тока и напряжения. Период колебаний составлял $0.6 \mu \mathrm{s}$, амплитуда тока и время его затухания соответственно $1 \mathrm{kA}$ и $1.2 \mu \mathrm{s}$. При этом на осциллограммах выделялись два характерных момента времени: появление напряжения на разрядном промежутке и пробой. За момент пробоя $t_{b d}$ был принят момент начала роста тока и соответственно спада напряжения.

Просвечивание разряда осуществлялось твердотельным лазером с длиной волны $532 \mathrm{~nm}$ и длительностью импульса на полувысоте $6 \mathrm{~ns}$.

В экспериментах использовалась оптическая схема на базе интерферометра Маха-Цендера. Изображение интерференционной картины, локализованной в области разрядного промежутка, строилось с помощью объектива с фокусным расстоянием $23 \mathrm{~cm}$ на фотокатоде электронно-оптического регистратора. Коэффициент увеличения равен десяти. Экспозиция кадра определялась длительностью импульса лазера.

Теневые изображения получались путем перекрытия опорного плеча интерферометра.
В экспериментах по фотографированию собственного свечения разряда лазер не использовался. Экспозиция кадра электронно-оптического регистратора составляла 40 ns. При фотографировании ранних стадий разряда (на временах менее $40 \mathrm{~ns}$ ) часть времени экспозиции опережала начало формирования разряда.

Сдвигом момента запуска лазера и электроннооптического регистратора относительно момента пробоя обеспечивалась визуализация различных стадий разрядного процесса. Съемка велась в однокадровом режиме один кадр за импульс. Разрешающая способность оптической системы составляла $5 \mu \mathrm{m}$ на три пикселя.

\section{3. Экспериментальные результаты и их обсуждение}

Фотографии собственного свечения разряда позволяют установить следующую его динамику: возникновение диффузного канала и катодных пятен, затем анодных пятен, последующее распространение этих светящихся образований навстречу друг другу, формирование ярко светящегося канала. Диаметры диффузного и ярко светящегося канала достигают соответственно 1 и $0.3 \mathrm{~mm}$. Размеры пятен составляют от 20 до $50 \mu \mathrm{m}$.

Формирование диффузного канала, катодных и анодных пятен происходит в интервале от 0 до $5 \mathrm{~ns}$, а распространение светящихся образований от пятен и замыкание промежутка - в интервале от 5 до $15 \mathrm{~ns}$ относительно появления напряжения на промежутке. Скорость распространения ярко светящегося канала от катода составляет порядка $10^{5} \mathrm{~m} / \mathrm{s}$.

Следует отметить, что описанная динамика свечения в целом аналогична обнаруженной ранее в подобных типах разрядов (см., например, [14]).

На рис. 3 представлены тенеграммы разряда для различных острийных электродов, а на рис. 4 - для основного электрода в различные моменты времени.

Видно, что для всех использованных электродов разряд развивается в микроканальной форме: канал пред- 
$a$
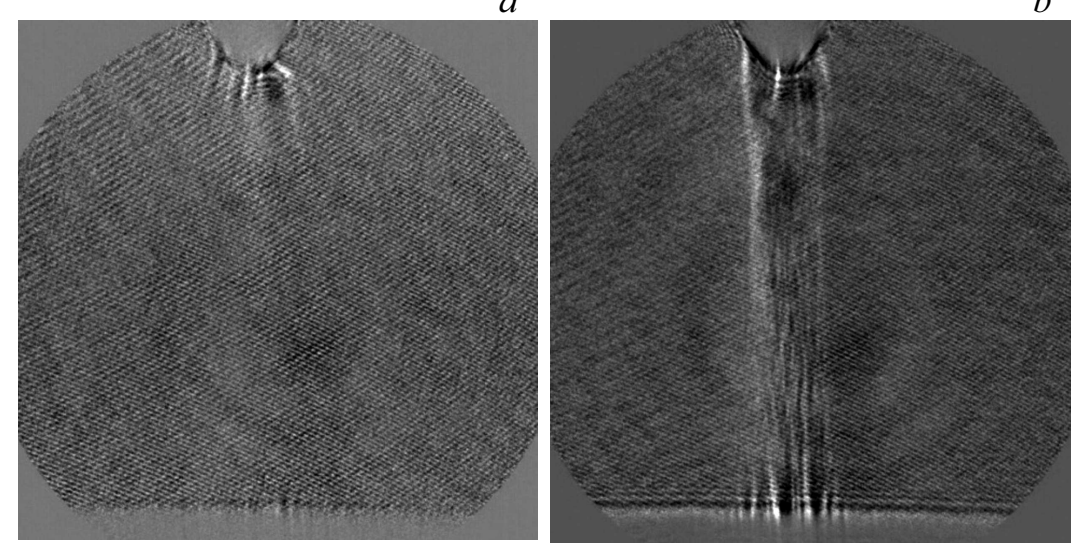

$b$
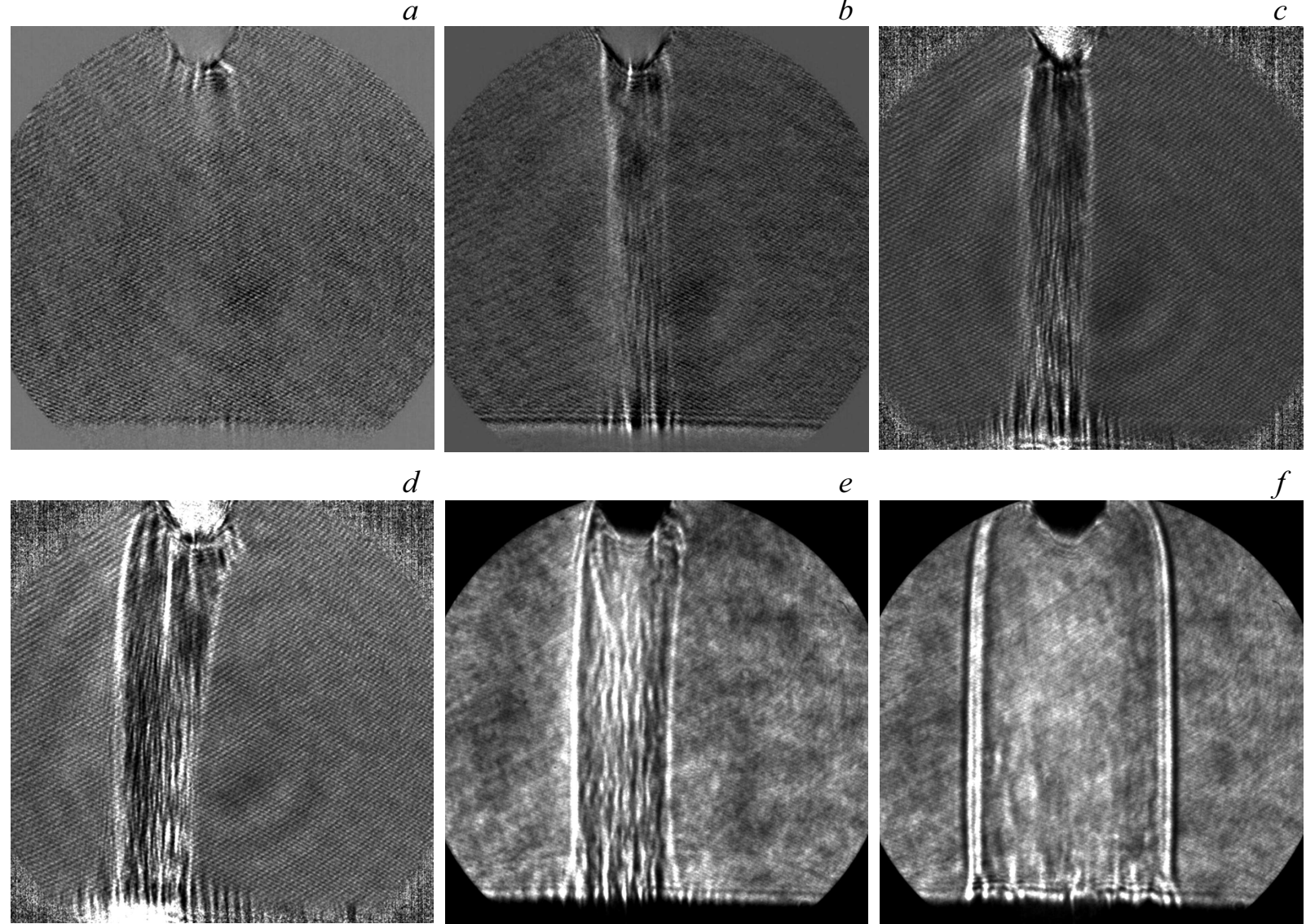

$e$
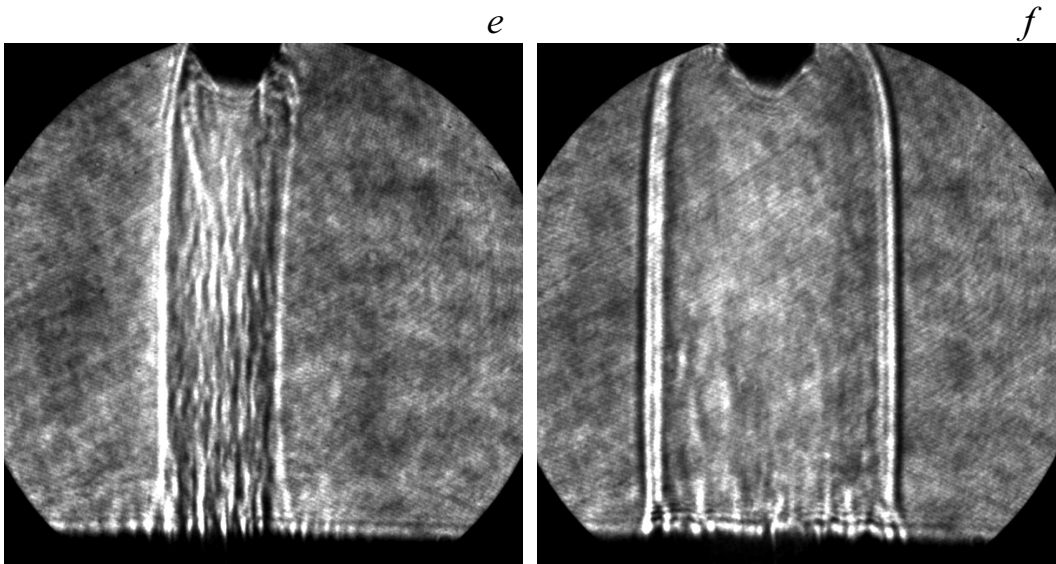

Рис. 4. Тенеграммы разряда в различные моменты времени относительно $t_{b d}: a-1, b-4, c-10, d-14, e-32, f-90 \mathrm{~ns}$. Электрод-острие находится сверху.

ставляет собой пучок большого числа микроканалов. Сразу после пробоя (рис. $4, a$ ) на тенеграммах регистрируются плазменные образования диаметром от 20 до $50 \mu \mathrm{m}$, развивающиеся от острийного электрода. Микроструктурированный канал замыкает промежуток к 2 ns и имеет форму, близкую к цилиндрической.

В течение 10-15 ns после пробоя ток разряда резко нарастает и достигает значения $1 \mathrm{kA}$. При этом до 15-20 ns диаметр канала почти не меняется и составляет около $250 \mu \mathrm{m}$, что свидетельствует об интенсивном росте проводимости плазмы в микроканалах в этот период. После этого начинается интенсивное радиальное расширение канала (рис. 4,e,f), на границе которого формируется ударная волна.

На рис. 5 представлены интерферограммы разряда в различные моменты времени относительно пробоя. Первое смещение полос носит отрицательный характер и регистрируется на $3 \mathrm{~ns}$ вблизи острия (рис. $5, a$ ) и на $5 \mathrm{~ns}$ вблизи плоского электрода (рис. $5, b$ ). В обоих случаях поперечные размеры областей искажения полос не превосходят $50 \mu \mathrm{m}$. Концентрация электронов в этих областях, оцененная по (6), составляет $\left\langle N_{e}\right\rangle \approx 4 \cdot 10^{19} \mathrm{~cm}^{-3}$. Появление указанных плазменных образований можно связать с формированием катодных и анодных пятен, регистрируемых на фотографиях собственного свечения разряда.

Начиная примерно с десятой наносекунды относительно пробоя, отрицательное смещение интерференционных полос регистрируется по всей длине канала.

К 15-30 ns канал разряда приобретает довольно четкую границу, после чего на ней регистрируется положительное смещение полос, что обусловлено появлением уплотненной, по сравнению с невозмущенным воздухом, оболочки - фронта ударной волны. Отметим, что вблизи острийного электрода положительное смещение регистрируется несколько раньше.

Следует особо отметить, что на интерферограммах также регистрируется микроструктура разряда, проявляющаяся в модуляции интенсивности интерференционных полос.

На рис. 6 представлены восстановленные по (3) распределения изменения показателя преломления по радиусу канала в различные моменты времени в центральной области промежутка.

Полученные зависимости демонстрируют динамику расширения канала, изменения концентрации плазмы, формирования и распространения ударной волны. Ска- 

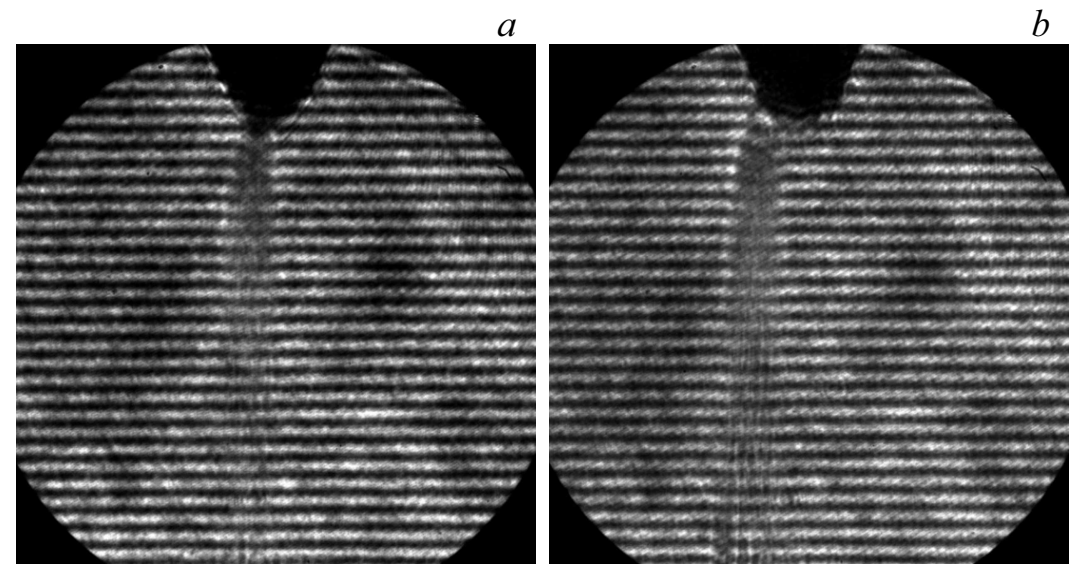

$b$
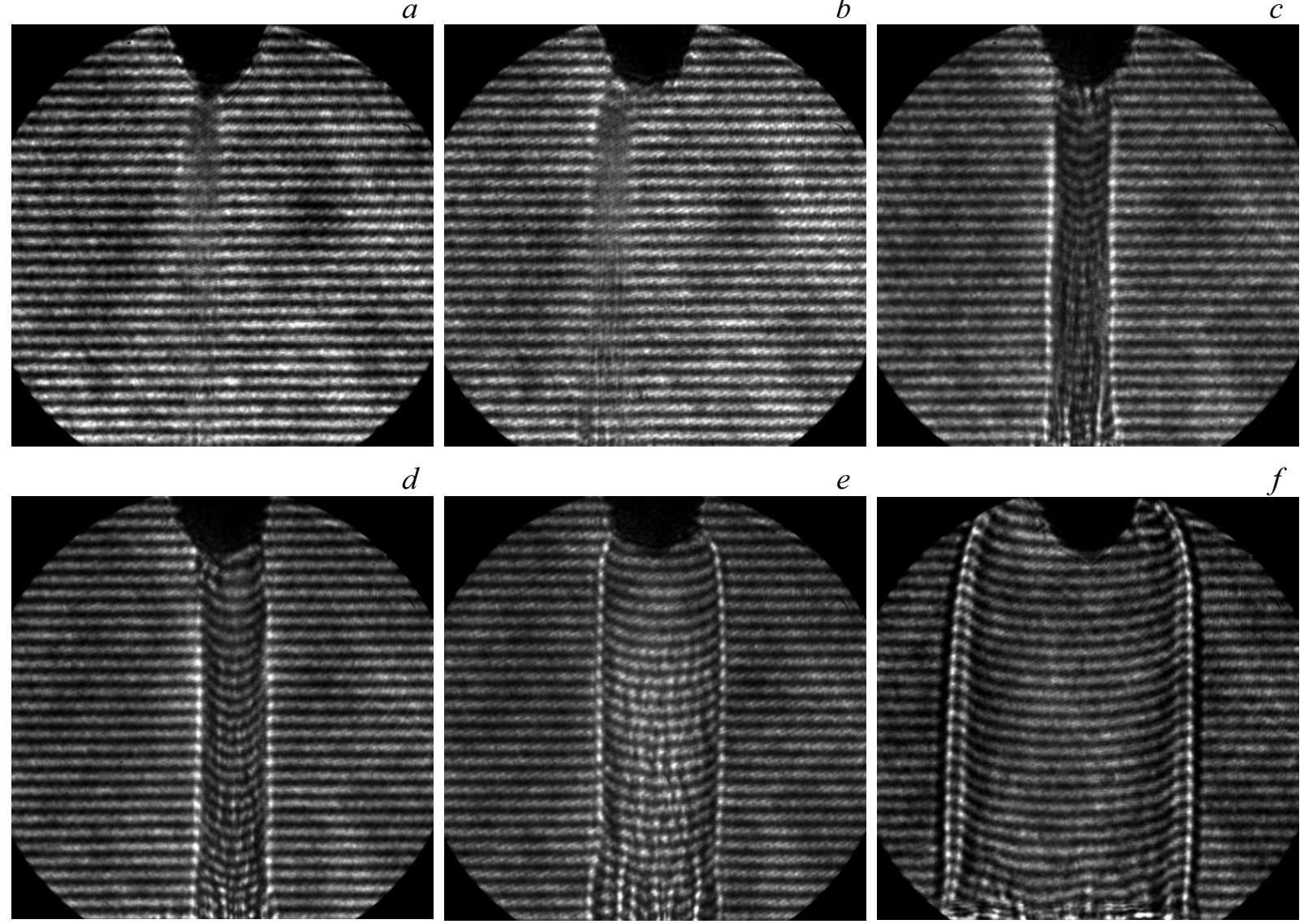

Рис. 5. Интерферограммы разряда в различные моменты времени относительно $t_{b d}: a-3, b-5, c-15, d-23, e-44$, $f-126 \mathrm{~ns}$. Электрод-острие находится сверху.

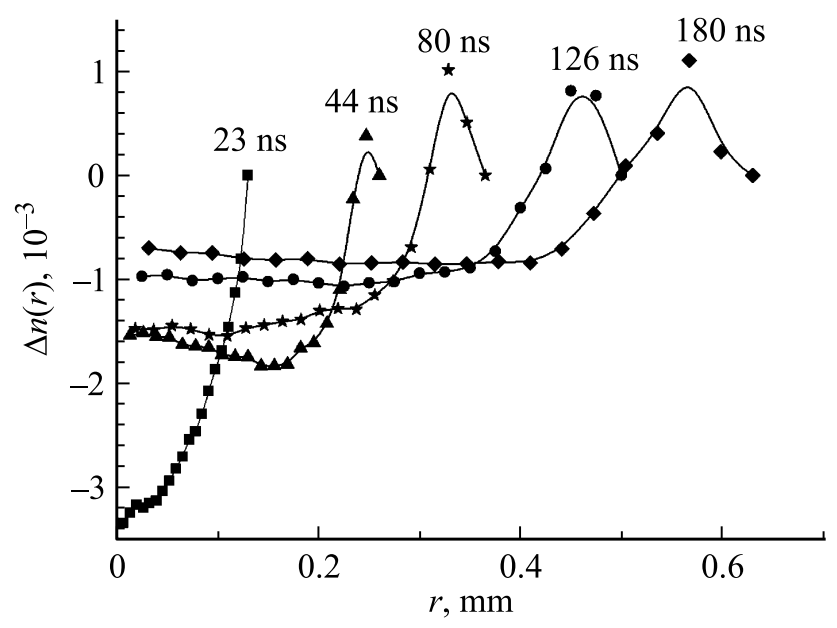

Рис. 6. Распределения изменения показателя преломления по радиусу канала в различные моменты времени относительно $t_{b d}$.

чок плотности в ударной волне равен, примерно, четырем.

Оценка зависимости средней концентрации электронов от времени в центральной области разрядного промежутка показывает, что максимальных значений концентрация достигает на временах 20-30 ns. Согласно рис. 6, до этого времени вкладом газодинамических процессов (изменением концентрации газа в канале) в изменение показателя преломления можно пренебречь. На рис. 7 представлено распределение концентрации электронов по радиусу канала, полученное по формуле (5) из приведенной на рис. 6 зависимости изменения показателя преломления для $23 \mathrm{~ns}$. Полученное распределение хорошо аппроксимируется функцией Гаусса.

Как видно из рис. 7, к двадцатой наносекунде в центре канала происходит полная однократная ионизация молекул воздуха. Если к этому моменту времени молекулы азота и кислорода полностью диссоциированы, то степень ионизации газоразрядной плазмы равна 50\%.

В более поздние моменты времени (рис. 6) происходит интенсивное расширение канала. Как видно из рис. 6 , внутри канала показатель преломления отрицательный и изменяется незначительно относительно своего минимального значения. Данная область характеризуется высокой концентрацией электронов и является токопроводящей. Эта область окружена некоторым переходным слоем (оболочкой), где $\Delta n$ плавно растет от своего минимального значения до нуля. Например, для $t=44 \mathrm{~ns}$ 
Результаты расчетов параметров искрового канала

\begin{tabular}{c|c|c|c|c|c|c}
\hline$t, \mathrm{~ns}$ & $-\Delta n, 10^{-3}$ & $r, \mathrm{~mm}$ & $N_{m}, 10^{19} \mathrm{~cm}^{-3}$ & $-\Delta n_{m}, 10^{-4}$ & $-\Delta n_{e}, 10^{-3}$ & $N_{e}, 10^{19} \mathrm{~cm}^{-3}$ \\
\hline 44 & 1.75 & 0.21 & 0.73 & 2.13 & 1.54 & 1.22 \\
\hline 81 & 1.34 & 0.3 & 0.36 & 2.53 & 1.09 & 0.86 \\
\hline 126 & 1.01 & 0.35 & 0.27 & 2.63 & 0.75 & 0.59 \\
\hline 180 & 0.84 & 0.4 & 0.22 & 2.7 & 0.57 & 0.45
\end{tabular}

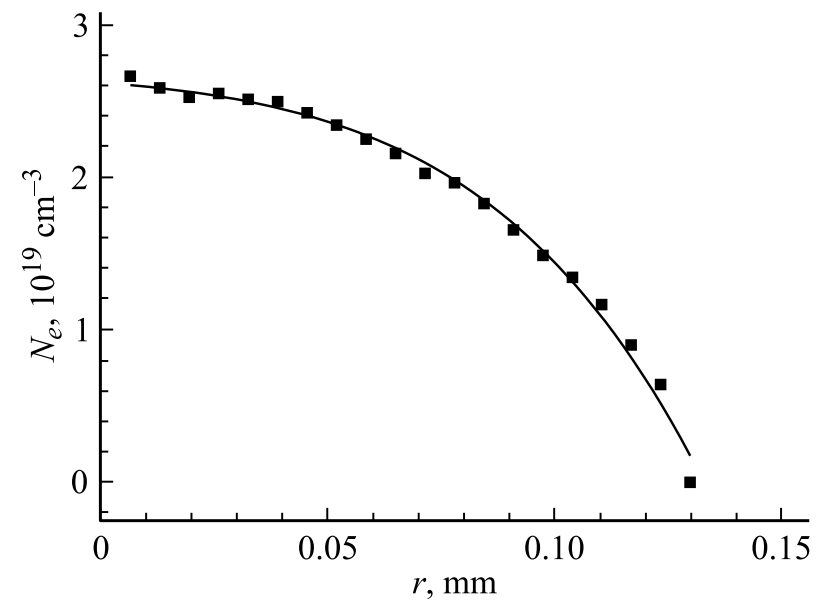

Рис. 7. Зависимость концентрации электронов от радиуса канала (точки) и аппроксимация функцией Гаусса (линия) для $23 \mathrm{~ns}$ относительно пробоя.

толщина этого слоя составляет $\sim 50 \mu \mathrm{m}$. Далее следует скачок уплотнения нейтрального газа (ударная волна).

Наличие внутренней высокопроводящей области, отделенной от фронта ударной волны оболочкой, подтверждается также сопоставлением фотографий собственного свечения, тенеграмм и интерферограмм.

Близкие результаты были получены в работах $[1,3]$, где впервые были обнаружены описанные выше структурные составляющие канала разряда: токопроводящая область, промежуточная оболочка и ударная волна, которые регистрировались после $100 \mathrm{~ns}$. В настоящих исследованиях формирование указанной структуры происходит несколько раньше - к 40-50 ns.

Тот факт, что в токопроводящей части канала концентрация плазмы по его сечению изменяется слабо, позволяет оценить состав плазмы в процессе разряда. Выполним соответствующие вычисления.

Пусть $r_{0}$ - радиус канала до момента начала его расширения $t_{0}, r$ - радиус канала в последующий момент времени $t$. Тогда концентрация молекул газа $N_{m}$ на этот момент времени определим как

$$
N_{m}=N_{0}\left(\frac{r_{0}}{r}\right)^{2},
$$

где $N_{0}=2.7 \cdot 10^{19} \mathrm{~cm}^{-3}-$ концентрация молекул воздуха при атмосферном давлении.
Изменение показателя преломления $\Delta n_{m}$ за счет расширения канала составит

$$
\Delta n_{m}=\left(n_{0}-1\right)\left[\left(\frac{r_{0}}{r}\right)^{2}-1\right] .
$$

Таким образом, определив $\Delta n_{m} \mathrm{e}$, можно из экспериментального значения $\Delta n$ выделить электроннуею составляющую изменения показателя преломления $\Delta n_{e}$ и определить концентрацию электронов $N_{e}$. Результаты расчетов по указанной процедуре с использованием распределений $\Delta n$, представленных на рис. 6 , сведены в таблицу. За начальный радиус канала выбрано значение $r_{0}=0.11 \mathrm{~mm}$, полученное для момента времени $t=17 \mathrm{~ns}$. В качестве радиуса токопроводящего канала принимался радиус переходного слоя.

Из сравнения значений концентраций электронов и молекул остаточного газа можно предположить, что, по крайней мере, на временах от 40 до $180 \mathrm{~ns}$, в канале происходит полная диссоциация молекул азота и кислорода с полной однократной ионизацией образовавшихся атомов.

Полученные результаты позволяют объяснить эффект, связанный с увеличением показателя преломления в центре канала (рис. 6), что особенно заметно на $44 \mathrm{~ns}$. Как видно из рис. 7, на начальной стадии разряда (23 ns) в центральной части канала концентрация электронов и соответственно плотность тока существенно выше, чем на периферии. Это приводит к большему нагреву ионизованного газа в данной области, что вызывает его перераспределение со снижением концентрации плазмы в центре и повышением на периферии.

На рис. 8 представлена определенная из интерферограмм зависимость скорости границы канала в различные моменты времени и ее линейная интерполяция по методу наименьших квадратов.

Использование линейной интерполяции вместо классического автомодельного решения для точечного взрыва в цилиндрической геометрии [2] $v \propto t^{-1 / 2}$ обусловлено несколькими причинами. Во-первых, в экспериментах данные получены в разных импульсах. Во-вторых, указанное автомодельное решение справедливо для мгновенного энерговыделения, сосредоточенного на оси, в то время как в разряде энерговклад является непрерывным и распределенным.

Полагая, что выделяющаяся в канале разряда энергия расходуется на нагрев газа, полученные данные по ско- 


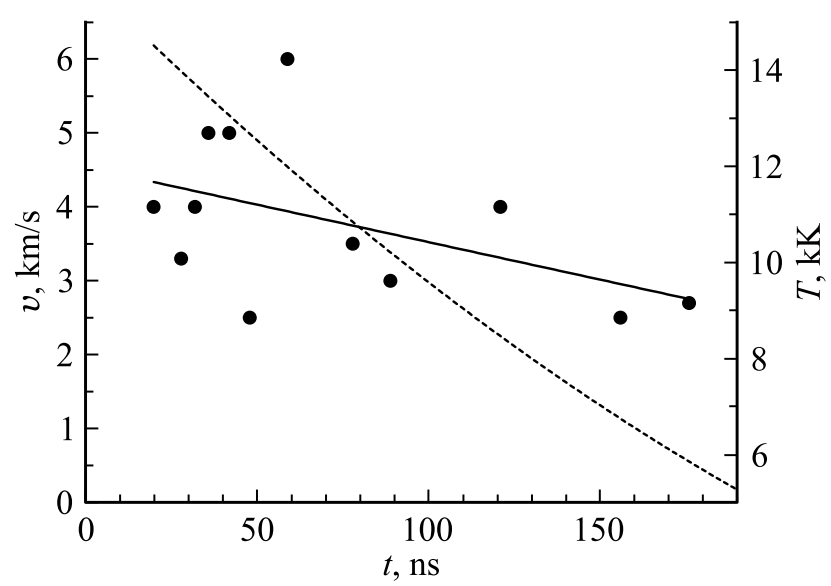

Рис. 8. Зависимости скорости расширения границы (точки определенные по интерферограммам значения, сплошная линия - линейная аппроксимация) и температуры (штриховая линия) канала от времени.

рости расширения канала позволяют оценить изменения температуры газа в канале, как [2]

$$
\Delta T=\frac{1}{\pi \alpha c_{f}} v^{2},
$$

где $c_{g}$ - удельная теплоемкость воздуха, $\alpha-$ численный коэффициент. На рис. 8 представлено изменение температуры канала во времени. При этом температура вычислялась с использованием указанной выше линейной зависимости скорости от времени.

Отметим, что для максимального значения скорости $6 \mathrm{~km} / \mathrm{s}$ (рис. 8), значение температуры, оцененное по (7), составляет около $30 \mathrm{kK}$.

Полученные значения температуры газа в канале, повидимому, допускают полную его ионизацию. Так, согласно [4], полная однократная ионизация атомов азота в канале искры происходит при температуре около $20 \mathrm{kK}$.

Следует особо отметить, что представленные в настоящей работе результаты измерений концентрации электронов и оценки температуры являются интегральными по сечению канала. Поскольку канал состоит из микроканалов, локализующих протекание тока, концентрация плазмы в них может быть выше представленных значений. Можно предположить, что, в некотором смысле, динамика каждого отдельного микроканала подобна развитию канала в целом, но на меньших масштабах.

Отметим, что близкие результаты по значениям концентрации электронов были получены в работах [5-7], где при схожих условиях реализации разряда также использовался интерференционный метод диагностики. Вместе с тем в этих работах не была обнаружена микроканальная структура разряда, что может быть связано с относительно низкой пространственной разрешающей способностью систем регистрации, используемых в [5-7].

Другой причиной может являться особенность построения изображения разряда в нашей оптической схеме, в которой регистратор находился на расстоянии более $2 \mathrm{~m}$ от объектива, что позволяло дискриминировать рассеянные на микронеоднородностях лучи зондирующего излучения и получать контрастное теневое изображение.

\section{Заключение}

Получены фотографии свечения, тенеграммы и интерферограммы искрового разряда в промежутке острие (катод)-плоскость при межэлектродном зазоре $1.5 \mathrm{~mm}$.

На фотографиях свечения условно выделены следующие последовательные стадии разряда:

- формирование диффузного канала, катодных и анодных пятен на временах 0-5 ns после появления напряжения на промежутке;

- прорастание плазменных образований от пятен и замыкание промежутка на временах 5-10 ns;

- формирование искрового канала.

Установлено, что на тенеграммах искровой разряд регистрируется в виде множества микроканалов, замыкающих разрядный промежуток. Микроканалы наблюдаются, начиная с первых наносекунд после пробоя при различных формах острийного электрода. Динамика разряда на временах от единиц до сотен наносекунд включает развитие микроканалов от острия вглубь разрядного промежутка, расширение микроканалов, формирование общего фронта ударной волны искры и его движение.

По интерферограммам определена динамика концентрации электронов в различных областях разрядного промежутка:

- раньше всего сдвиг интерференционных полос регистрируется на $3 \mathrm{~ns}$ вблизи острия и на $5 \mathrm{~ns}$ вблизи плоского электрода в областях с поперечным размером менее $50 \mathrm{~mm}$, где оценка концентрации электронов дает величину $\approx 4 \cdot 10^{19} \mathrm{~cm}^{-3}$;

- максимальная электронная концентрация достигается на оси канала разряда на временах 20-30 ns и составляет $\approx 2.6 \cdot 10^{19} \mathrm{~cm}^{-3}$;

- к 40-50 ns происходит формирование следующих структурных составляющих канала разряда: токопроводящая область, промежуточная оболочка и ударная волна;

- на временах от 40 до 180 ns в канале, по-видимому, происходит полная диссоциация молекул азота и кислорода с полной однократной ионизацией образовавшихся атомов.

Определена скорость радиального расширения канала, которая в интервале времени от 20 до $200 \mathrm{~ns}$ изменяется от 6 до $2.5 \mathrm{~km} / \mathrm{s}$. Скачок плотности в ударной волне по отношению к невозмущенному воздуху равен, примерно, четырем.

Выполнена оценка температуры газа в канале искры: на временах от 20 до $200 \mathrm{~ns}$ температура изменяется в среднем от 15 до $5 \mathrm{kK}$. 


\section{Благодарности}

Авторы благодарны В.И. Карелину за полезное обсуждение и высказанные замечания при подготовке статьи.

\section{Конфликт интересов}

Авторы заявляют, что у них нет конфликта интересов.

\section{Список литературы}

[1] И.С. Абрамсон, Н.М. Гегечкори, С.И. Драбкина, С.Л. Мандельштам. ЖЭТФ, 17 (10), 862 (1947).

[2] С.И. Драбкина. ЖЭТФ, 21 (4), 473 (1951).

[3] Н.М. Гегечкори. ЖЭТФ, 21 (4), 493 (1951).

[4] С.Л. Мандельштам, Н.К. Суходрев. ЖЭТФ, 24 (6), 701 (1953).

[5] Е.В. Паркевич, С.И. Ткаченко, А.В. Агафонов, А.Р. Мингалеев, В.М. Романова, Т.А. Шелковенко, С.А. Пикуз. ЖЭТФ, 151 (4), 627 (2017).

[6] Е.В. Паркевич, А.И. Хирьянова, А.В. Агафонов, С.И. Ткаченко, А.Р. Мингалеев, Т.А. Шелковенко, А.В. Огинов, С.А. Пикуз. ЖЭТФ, 153 (3), 504 (2018).

[7] Jie Huang, Lin Yang, Hongchao Zhang, Lei Chen, Xianying Wu. Chin. Phys. B, 28 (5), 055202-1 (2019).

[8] К.И. Алмазова, А.Н. Белоногов, В.В. Боровков, Е.В. Горелов, И.В. Морозов, А.А. Тренькин, С.Ю. Харитонов. ЖТФ, $88(6), 823$ (2018).

[9] К.И. Алмазова, А.Н. Белоногов, В.В. Боровков, Е.В. Горелов, И.В. Морозов, А.А. Тренькин, С.Ю. Харитонов. ЖТФ, 89 (1), 69 (2019).

[10] К.И. Алмазова, А.Н. Белоногов, В.В. Боровков, Е.В. Горелов, И.В. Морозов, А.А. Тренькин, С.Ю. Харитонов. ЖТФ, 89 (4), 512 (2019).

[11] А.А. Тренькин. ЖТФ, 89 (2), 189 (2019).

[12] М.М. Скотников. Теневые количественные методы в газовой динамике. (Наука, М., 1976).

[13] Л.А. Душин, О.С. Павличенко. Исследование плазмы с помощью лазеров. (Атомиздат, М., 1968).

[14] Е.Х. Бакшт, О.М. Блинова, М.В. Ерофеев, В.И. Карелин, В.С. Рипенко, В.Ф. Тарасенко, А.А. Тренькин, Ю.М. Шибитов, М.А. Шулепов. ФП., 42 (9), 859 (2016). 